\section{Perspektiven für ein DRG-System Investitionsstau erfordert Nachjustierung}

\begin{abstract}
Dr. Manfred Georg Krukemeyer lenkt als Vorsitzender der Gesellschaftsversammlung seit 1994 die Geschicke der Paracelsus-Kliniken Deutschland. Der Grundstein für die Klinikkette wurde 1968 in Osnabrück gelegt. Heute beschäftigt der Konzern bundesweit $\mathbf{5 0 0 0}$ Mitarbeiter in 17 Akutkrankenhäusern, 11 Reha-Kliniken und 9 ambulanten Einrichtungen. Sie versorgen rund 100000 stationäre Patienten pro Jahr. Die akut-stationären Patienten verweilen im Durchschnitt 6,5 Tage im Krankenhaus, d.h. etwa einen Tag weniger als Patienten in anderen privaten oder öffentlichen Krankenhäusern.
\end{abstract}

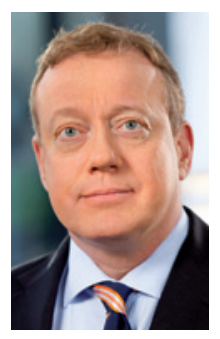

? Herr Dr. Krukemeyer, lässt sich die niedrige Verweildauer in Ihren Kliniken durch das pauschalisierte Entgeltsystem erklären?

Dr. Manfred Krukemeyer: Zum Teil, ja. Dieses hatte die Optimierung der BehandlungsabDr. Manfred Georg läufe sowie eine bessere VerKrukemeyer zahnung von ambulanter und stationärer Versorgung sowie der anschließenden Reha zur Folge. Andererseits können natürlich auch der medizinische Fortschritt sowie ein Bewusstseinswandel dafür verantwortlich gemacht werden.

\section{Bewusstseinswandel? Inwiefern?}

Krukemeyer: Nun, kürzere Verweildauern entsprechen für einen großen Teil der Patientinnen und Patienten auch deren Bedürfnissen. Da sich Krankenhäuser zunehmend als Dienstleister verstehen, kommen wir dem im medizinisch vertretbaren Rahmen auch nach. In Amerika sind die Verweildauern übrigens immer noch deutlich kürzer als bei uns. Ich sage nicht, dass wir da unbedingt hinkommen müssen, es hilft aber, wenn man die Perspektive erweitert und sich anschaut, wie andere Länder bestimmte Dinge angehen. Den Kostenträgern in Deutschland sind die Verweildauern heute anscheinend immer noch zu lang. Mittlerweile werden durchschnittlich 7,5\% aller Krankenhausfälle durch den MDK hinsichtlich der unteren oder oberen Grenzverweildauer strittig gestellt. Das bedeutet, dass diese Fälle nach Ansicht der Krankenkassen noch zu lange im Krankenhaus gelegen haben. Die Kostenträger möchten die Verweildauer noch weiter kürzen. Dies wird aber ohne den gesamtgesellschaftlichen Konsens nicht gehen.

\section{? Welche Auswirkungen hat das DRG-System für die Arbeit auf den Stationen?}

Krukemeyer: Die Organisation „Krankenhaus" hat sich in den vergangenen Jahren deutlich verändert. Jede Einrichtung hat auf die eine oder andere Art an der Effizienzsteigerung gearbeitet. Personalreduktionen, Fusionen, Zentralisierungen, Standardisierungen, Outsourcing sind da nur einige Stichwörter. Das alles waren und sind Prozesse, die innerhalb und auBerhalb eines Hauses erhebliche Wellen geschlagen haben. Ich meine, die allermeisten Krankenhäuser haben hier ihre Hausaufgaben gemacht. Mehr ist da kaum zu holen - denn anders als in anderen Branchen müssen wir ja stets im Blick haben, dass unser Geschäft die Gesundheit der Menschen ist, die uns vertrauen. Wir sind für kranke Menschen verantwortlich.

? Ein Ziel bei der Einführung der DRG ausgaben. Inwieweit ist dieses Ziel erreicht worden?

Krukemeyer: Zunächst einmal bin ich mir gar nicht sicher, ob die Reduktion der Ausgaben tatsächlich ein Ziel war. Eigentlich sollte doch ein gerechteres, pauschaliertes Vergütungssystem eingeführt werden.

\section{? Ist das aus ihrer Sicht gelungen?} Krukemeyer: Tja... einerseits gab es Einsparmöglichkeiten. Und wie gesagt, diese haben die meisten Häuser auch genutzt. Aber andererseits kostet der medizinische Fortschritt auch Geld. Und zwar viel Geld. Es ist großartig, dass wir heute über 70-Jährigen weitgehend risikofrei eine neue Hüfte implantieren können. Es ist toll, dass wir immer mehr minimalinvasiv machen können. Aber das ist doch war die Reduktion der Krankenhaus- nicht zum Nulltarif zu haben! Gleichzeitig steigen die Erwartungen der Menschen, dass solche Operationen auch durchgeführt werden. Gerade wenn es um die Gesundheit geht, will doch jeder Mensch am Fortschritt teilhaben. Und das bedeutet einen erhöhten Investitionsaufwand, den wir heute kaum noch abdecken können.

\section{Welche positiven Effekte hat das} pauschalisierte Entgeltsystem?

Krukemeyer: Wie gesagt: Es ist durchaus positiv, dass Krankenhäuser quasi zu Kooperationen, Standardisierungen etc. gezwungen wurden. Aber diese strukturellen Wirtschaftlichkeitsreserven sind nun aufgebraucht. Ab jetzt geht es ans Eingemachte, ab jetzt kann nur noch über Veränderungen in der Organisation nachgedacht werden. Zum Beispiel sind sinnvolle Kürzungen in den Verweildauern oder gerechtere Verteilung der finanziellen Ressourcen im System, Schaffung von Transparenz denkbar. Das alles braucht aber Zeit und Geld. Und nicht zuletzt: Dafür muss ein Umdenken in den Köpfen der Menschen stattfinden. Und das ist nicht trivial.

\section{Wo sehen Sie Fehlentwicklungen?} Krukemeyer: Das System ist wirtschaftlich getriggert. Zumindest kann und wird es - auch wenn das möglicherweise nicht intendiert war - von den Häusern so ausgelegt. Was bleibt manchen Häusern anderes übrig, als genau die Leistungen anzubieten, die besonders lukrativ sind? Da bleiben Leistungen aus der Grund- und Regelversorgung leider häufig auf der Strecke. Momentan wird ja häufig darüber debattiert, dass Ärztinnen und Ärzte nur operieren, um die Leistung zu steigern. Davon, dass Patientinnen und Patienten häufig bestimmte OPs einfordern, redet kein Mensch. Und auch nicht davon, dass eine OP ja immerhin von mehreren Ärzten - dem Einweisenden und dem Operateur - befürwortet werden muss. Und nicht zuletzt: Wer ohne Notwendigkeit operiert, macht sich strafbar. Hier wird momentan hitzig diskutiert. Ein wenig mehr Sachlichkeit täte der Diskussion gut.

\section{Sehen Sie einen Ausweg aus dem} Kreislauf der Mengensteigerung? Krukemeyer: Zunächst einmal: Nicht jede Mengensteigerung ist negativ zu bewerten. Denken Sie an den erwähnten medizinischen Fortschritt oder die demografische 
Entwicklung sowie die geänderten Bedürfnisse der Menschen. Diese Faktoren haben Mengensteigerungen zur Folge. Aber wir müssen zwischen sinnvoller und künstlich produzierter Mengensteigerung unterscheiden. Die Fallpauschalen müssten den Investitionsbedarf decken. Und abseits aller Zahlen muss auf breiter Ebene darüber ehrlich diskutiert und im Anschluss konsentiert werden, was wir uns für die Gesundheitsversorgung der Menschen in Deutschland wünschen. Und was wir bezahlen wollen und können.

\section{? Hat das DRG-System eine Zukunft} oder sehen Sie Bedarf für ein alternatives Finanzierungssystem?

Krukemeyer: Eigentlich ist doch nicht das DRG-System unser Problem, sondern die dualistische Krankenhausfinanzierung. Durch diese Finanzierung werden die Betriebskosten über die DRG-Einnahmen und somit durch die Krankenkassen gezahlt. Die Investitionskosten hingegen werden durch die Länder finanziert. Aber hier ist es mittlerweile zu einem so großen Investitionsstau gekommen, dass viele Häuser mit den pauschalen Fördermitteln ihre Ausgaben auf diesem Sektor nicht mehr decken können. Was machen diese Häuser? Sie subventionieren quer aus einem anderen Topf, nämlich den DRG-Einnahmen. Das kann auf Dauer nicht gut gehen. Die Steigerungen in der DRG-Vergütung decken zum Großteil ja nicht einmal die Inflationsrate ab. Das DRG-System muss vielleicht nachjustiert werden. Was verändert werden muss, ist die Krankenhausfinanzierung.

\section{? Die Paracelsus-Kliniken sind aus} einer Praxisklinik hervorgegangen. Wie müsste sich die Verzahnung von ambulanter und stationärer Versorgung unter dem Aspekt der Finanzierung künftig gestalten?

Krukemeyer: Ambulante und stationäre Versorgung unterliegen grundsätzlich verschiedenen Finanzierungssystemen. Hier müsste eine Anpassung oder Vereinheitlichung der Systeme stattfinden. Zumal diese Verzahnung ja nun wirklich im Sinne der Patientinnen und Patienten ist. Denkbar wäre beispielsweise ein Modell ähnlich der integrierten Versorgung mit einer Komplexpauschale. Ambulanter und stationärer Sektor erhalten beide eine Vergütung für die Behandlung eines Patienten innerhalb eines bestimmten Behandlungskorridors.

\section{? Ihr Fazit?}

Krukemeyer: Der Bettenabbau generell wird weitergehen, aber die hohe medizinische Qualität wird bleiben.

Herr Dr. Krukemeyer, wir bedanken uns für das Gespräch!

Das Interview führte Dr. Adelheid Weßling, Düsseldorf. 